\title{
The Hippocampus, Objects, and Their Contexts
}

\author{
Helen J. Cassaday \\ University of Nottingham
}

\author{
J. N. P. Rawlins \\ University of Oxford
}

\begin{abstract}
Rats with hippocampal aspiration lesions and controls were trained on delayed nonmatching to sample with small complex goal boxes, presented trial uniquely. A series of experiments then used pairs of large or small boxes, presented repeatedly. The lesions impaired choice accuracy when the rats were tested with large empty boxes but not when small boxes containing 3-dimensional objects were used. There was a comparable impairment when the rats were tested with pairs of large complex boxes, which contained arrays of objects, identical to those used in the smaller boxes but necessarily spaced further apart. Subsequent experiments revealed that the lesion deficit with large boxes was reduced by insertion of a continuous line of distinctive objects and eliminated by trial-unique presentation of large boxes. The results are discussed in terms of (non) spatial accounts of hippocampal function and the compensatory effects of novel object cues. We conclude that, for hippocampal rats, spatial cues, although useless, can nonetheless be profoundly disruptive.
\end{abstract}

There is general consensus that the hippocampal formation is essential for normal memory processing, but still disagreement as to the best theoretical account of its function. One clear distinction is between theorists advocating an exclusively spatial role for the hippocampal formation in learning and memory (Nadel, 1991; O'Keefe \& Nadel, 1978; Worden, 1992) and those preferring a nonspatial account (Eichenbaum, Otto, \& Cohen, 1994; Olton, Becker, \& Handelmann, 1979; Rawlins, 1985; Sutherland \& Rudy, 1989, 1995).

A series of studies from this laboratory suggests that the reliable demonstration of nonspatial working memory deficits after hippocampal damage depends on the circumstances under which the rats are tested (Cassaday \& Rawlins, 1995; Rawlins, Lyford, Seferiades, Deacon, \& Cassaday, 1993; Yee \& Rawlins, 1994). The earliest of these studies used a discrete-trial delayed matching to sample (DMS) design, with small goal boxes containing complex objects (Aggleton, 1985) or larger and plainer goal boxes that differed mainly in their surfaces' paint finish and texture (Raffaele \& Olton, 1988). The results showed that a lesion-induced impairment was present only (a) when the small complex stimuli were used repeatedly within sessions, rather than in a pseudo-trial-unique (PTU) design, and (b)

Helen J. Cassaday, Department of Psychology, University of Nottingham, Nottingham, England; J. N. P. Rawlins, Department of Experimental Psychology, University of Oxford, Oxford, England.

This research was supported by the Wellcome Trust Project Grant 036159, with additional support from the Medical Research Council Centre for Brain and Behaviour, and the McDonnell-Pew Foundation for Cognitive Neuroscience. We thank Greg Daubney for his excellent work on the histology, and we thank Bryan Coyle for building and decorating the goal boxes. Rob Deacon gave invaluable advice on the running of the $Y$-maze.

Correspondence concerning this article should be addressed to Helen J. Cassaday, Department of Psychology, University of Nottingham, University Park, Nottingham NG7 2RD England. Electronic mail may be sent via Internet to helen.cassaday@ nottingham.ac.uk. that this impairment was greatest when the stimuli that were used repeatedly were the large, plain ones. Yee and Rawlins used a delayed nonmatching to sample (DNMS) design and showed an impairment when large, plain goal boxes were used in a design in which repetition was not a factor because there was only one trial per day. Neither the matching rule (DMS, DNMS) nor the lesion method (hippocampal aspiration, fornix section) in use appeared to be critical determinants of the hippocampal deficit (Rawlins et al., 1993; Yee \& Rawlins, 1994).

A series of DMS experiments (Cassaday \& Rawlins, 1995) subsequently demonstrated that fornix-section impaired choice accuracy whenever rats were tested repeatedly, with large, empty goal boxes but not with small empty boxes of otherwise identical construction. This dissociation suggested a critical role for goal-box size, independent of box complexity. Repetition was again not a critical variable (cf. Yee \& Rawlins, 1994), and a preliminary experiment suggested that the lesion-induced impairment could not be overcome by adding familiar objects to (the choice ends of) the large goal boxes.

There is thus overwhelming evidence that hippocampectomized rats are impaired on DMS or DNMS when larger boxes are used as stimuli but are unimpaired when smaller boxes are used. Moreover, small boxes can support good performance whether or not they are used repeatedly and whether or not they themselves contain discrete objects. However, we cannot yet conclude that the use of large goal boxes will inevitably lead to lesion-induced deficits, regardless of whether or not they contain discrete objects as additional stimuli and regardless of whether the boxes are used repeatedly or in a PTU design. These issues have simply not been addressed experimentally to date.

The present study was therefore designed to establish whether the relative disadvantage with larger boxes might be reduced under certain circumstances. First, we provided more cues in larger boxes (in Experiments 2 and 3) and, in the final experiment, used a PTU schedule with a set of large boxes. We provided additional cues by inserting three- 
dimensional objects. These were sometimes identical to those used in small boxes for an additional control comparison within the same experiment (although in the larger boxes the same number of objects were necessarily spaced further apart). The objects were fixed either throughout the goal boxes or in a spatially contiguous series down one side. In addition, the series could be made up of identical (repeating) objects (e.g., a continuous line of toy pigs) or different (and distinctive) objects (e.g., a plastic dog placed next to a toy figure, followed by a bird, a pair of lion cubs, a toy soldier, and finally a polar bear). The goal boxes containing series of objects were designed explicitly to provide an "associative bridge" to test our earlier hypothesis that the "associative distance" from the choice point of the point of reinforcement in the larger boxes might be critical (Cassaday \& Rawlins, 1995; Introduction to present Experiment 3). We also included paint decoration (like that used in smaller boxes) in large complex (object) boxes. This decoration was provided by differences in pattern and color finish.

Large empty boxes provide diffuse cues like those typically provided by contexts. It is also clear that large boxes are contexts in a functional sense when objects are enclosed (see Nadel \& Willner, 1980). Throughout we refer to these boxes as "contexts," synonymous with extended spatial arrays or environments, but we also go on to consider the possibility that they are encoded in the same way as the contextual stimuli used in other experimental paradigms.

We compared performance with different sorts of box type in a mixed design: between subjects (in lesioned and nonlesioned groups) and within subjects as we tested rats with all box types (on different days) at each stage of the experimental series. The experimental schedule was as follows: We first gave rats limited preoperative training on DNMS with large empty goal boxes, presented repeatedly. We then allocated them to lesion and control conditions. Postoperatively, we trained them using small complex boxes presented trial-uniquely in Experiment 1 . We then tested the rats briefly with (a) repeated small and (b) repeated large goal boxes before the main series of experiments began.

First, in Experiment 2C, we compared DNMS performance under 4 conditions, run in rotation. In each condition we used a different pair of goal boxes: (a) small complex boxes, containing objects and paint decoration; (b) large complex boxes, with paint decoration and objects identical to those used in the small boxes in Condition 1; (c) large complex boxes, with paint decoration and objects different to the above; and (d) large empty boxes. Members of a pair were similar in kind but discriminably different from each other.

Second, in Experiment 3, we again inserted objects in large goal boxes but now as a continuous line rather than in a random array. There were again four conditions in which we used four pairs of boxes: (a) small boxes, each containing a line of identical objects; (b) large boxes, each containing a line of identical objects, the same as those used in the boxes in Condition 1; (c) large boxes, each containing a line of distinctive objects; and (d) large empty boxes.

Finally in Experiment 4, we tested the rats on DNMS without repetition of the stimuli within the daily session (i.e., on a PTU schedule). This condition used all of the large stimuli designed for the preceding experiments and a number of new ones, all decorated distinctively.

Thus the experiments aimed to further clarify the critical stimulus features that make nonspatial working memory tasks (in)soluble for rats with hippocampal lesions by determining the generality of the deficit with large goal-box stimuli. Specifically, we investigated whether this deficit might be overcome (a) by placing discrete objects into large boxes, thereby increasing their nonspatial cue content; (b) by arranging discrete objects into continuous lines within large boxes, to provide a nonspatial "associative bridge" between the choice point and the point of reinforcement; and (c) by decreasing possible sources of interference by reducing the repetitive use of the boxes.

The experiments reported below are intended to address the general issue of how best to describe the psychological consequences of conventional hippocampal system lesions. The further question of how to identify the critical neural substrate where the damage underlies these effects would require the assessment of rats with selective cytotoxic lesions; the present study does not address that issue.

\section{General Method}

\section{Subjects}

The subjects were 24 experimentally naive male Dark Agouti (DA) rats obtained from OLAC (Harlan UK LTD, Shaw's farm, Blackthorn, Bicester), weighing $180-220 \mathrm{~g}$ at the beginning of preoperative training, $155-195 \mathrm{~g}$ at surgery, $205-250 \mathrm{~g}$ after postoperative recovery, and then maintained at $230-275 \mathrm{~g}$ for the duration of the experiments. Two rats (one lesioned and one sham operated) died under anesthesia, so the experiments below were carried out on 22 rats.

The rats were caged in pairs preoperatively, but they were caged singly postoperatively to allow better weight control. Each rat received $10-20 \mathrm{~g}$ of rat chow (Witham, Essex, UK) upon the completion of the day's testing, except for a 2-week postoperative recovery period during which they were maintained on full food. Thereafter, the amount of food was individually adjusted for each rat to allow appropriate weight gain. Access to water was unlimited throughout. The rats were housed in a temperature-controlled room $\left(23^{\circ} \mathrm{C}\right)$, on a 12-hr light-dark cycle (lights on at 7:00 a.m.). All experiments were run during the light phase.

\section{Surgery}

All 24 rats were matched for preoperative performance scores and weight and allocated to one of three surgical groups: hippocampal aspiration (HIPP, $n=10$ ), cortical aspiration (CORT, $n=6$ ), and sham operated (sham, $n=8$ ). The day before surgery took place, the rats were given $0.05 \mathrm{ml}$ i.m. Vitamin $\mathrm{K}$ (Konakion: Roche, phytomenadione $2 \mathrm{mg} / \mathrm{ml}$ ). All subjects were anesthetized with an intraperitoneal injection of Avertin in a dose of $10 \mathrm{ml} / \mathrm{kg}$. (Avertin concentrate consists of $100 \mathrm{~g}$ of 2,2,2-tri-bromo-ethanol dissolved in $62 \mathrm{ml}$ of tertiary amyl alcohol: $1.25 \mathrm{ml}$ of this concentrate are added to $5 \mathrm{ml}$ absolute alcohol and $62.5 \mathrm{ml}$ of $0.9 \%$ saline [0.9\% wt/vol.]). The rats' scalps were shaved, and they were placed in a stereotaxic headholder. An incision was made in the scalp to expose the skull. A D-shaped hole was drilled on each side to reveal the dura covering the cortex overlying the dorsal hippocampus. The CORT and HIPP rats were transferred to a 
specially designed headholder (Rawlins \& Bennett, 1980) for aspiration lesions performed under visual control using a Wild M650 operating microscope (Heerbrugg, Switzerland). In the HIPP group, the dura was sectioned, and sufficient neocortex was removed to allow the hippocampus to be aspirated. The CORT group had an equivalent area of neocortex and corpus callosum aspirated to expose the alveus of the hippocampus. Both groups had the wound packed with Sterispon absorbable gelatin foam that had been soaked in sterile physiological saline. The dura was, as far as possible, drawn back over the wound.

All groups had sulphonamide powder (Acramide: Willington Medicals, Ltd., Shrewsbury, Shropshire) sprinkled over the exposed tissue, and the scalp was then sutured. All subjects were given 40,000 units of penicillin, $0.1 \mathrm{ml}$ i.m. (Bicillin: Brocades Great Britain Ltd., West Byfleet, Surrey, UK) before being returned to the home cage. One sham rat and one HIPP rat died without recovering from anesthetic, leaving group sizes as follows: HIPP, $n=9 ;$ CORT, $n=6$; sham, $n=7$.

\section{Histology}

At the end of Experiment 5, all the rats in the CORT and HIPP groups were given an overdose of sodium pentobarbitone and perfused through the heart with saline $(0.9 \% \mathrm{wt} / \mathrm{vol})$ followed by $10 \%$ formol-saline. The brains were removed, blocked, and stored in $30 \%$ sucrose buffered formalin for 4-5 weeks. They were then embedded in Cryo-M-Bed, frozen in iso-pentane over solid $\mathrm{CO}_{2}$, and sectioned in the coronal plane at $25-\mu \mathrm{m}$ using a cryostat. Every eighth section through the lesion was retained and stained with cresyl violet. The sections were inspected microscopically to assess the extent of the lesions. We report assessments at an anterior, an intermediate, and a posterior plane of sectioning, corresponding approximately to Plates 32,38 , and 43 in the atlas of Paxinos and Watson (1986).

\section{Apparatus and Maze Equipment}

The two-choice maze. This apparatus was developed from that used by Raffaele and Olton (1988) for use in the Rawlins et al. (1993) study. It was constructed of sheet aluminum alloy, comprising a start area that expanded into two goal areas. The walls of the apparatus were $20.3 \mathrm{~cm}$ high. The start area and each of the goal areas were covered by hinged Plexiglas lids; there was a hole above each goal area through which reward pellets (two 45-mg Noyes reward pellets) could be dropped. The start area measured $13.5 \mathrm{~cm}$ wide and $27.9 \mathrm{~cm}$ long, and the end nearest the goal arms could be closed with a guillotine door. At the end of the start area, where the maze opened into the two goal areas (total width, $27.8 \mathrm{~cm}$ ), there was an enclosed trapezoid space, the choice area, in which the rat made its selection between the goal arms. The two goal areas, each $13.9 \mathrm{~cm}$ wide and $33.2 \mathrm{~cm}$ long, lay side by side and were separated by a common wall. They were open at both ends. The end facing the start area could be closed with a guillotine door. The other open end allowed for the insertion of the removable goal boxes. Each goal area was illuminated by three 2.8 -W incandescent bulbs mounted on the outside of the Plexiglas lids. The apparatus rested on a table in a brightly lit room.

The $Y$-maze. This was the three-arm maze used in the Rawlins et al. (1993) study, developed from that used by Aggelton, Hunt, and Rawlins (1986), fully described in Steele and Rawlins (1989). It had three converging uniform arms. Each consisted in a $15-\mathrm{cm}$ long runway separated by a guillotine door from a $20-\mathrm{cm}$ long goal area. The goal boxes were inserted at the goal ends of the arms. The arms were enclosed with $20-\mathrm{cm}$ high aluminium sides, and the top of the maze was covered with Plexiglas lids. The goal areas were lit by three $2.8-\mathrm{W}$ bulbs mounted in the Perspex cover. Where the three runways converged, a trefoiled guillotine door separated each arm from the others. The testing room was otherwise the same as that described above.

\section{Stimuli}

Overview of stimuli. The large and small boxes differed in construction in two general respects. First, they were made of different materials: plywood versus hardboard, respectively. Second, they differed in overall size and in details of shape at the back and the sides. Other differences between the boxes arise from the systematic manipulation of the box complexity. The different box types are described in the Method sections for each experiment at the point of introduction; see Figure 1 for illustrations. Throughout, box "decoration" refers to differences in colored paint design. The objects placed in the different sorts of object box were plastic toys, nuts and bolts, and other small household tools or fittings. Table 1 provides a summary of box type abbreviations and conditions of presentation in the different experiments.

Large black or white empty goal boxes (LARGE-B/W-EMPTY). Large empty goal boxes were modeled after those of Raffaele and Olton (1988) for use in preoperative training (and Experiments
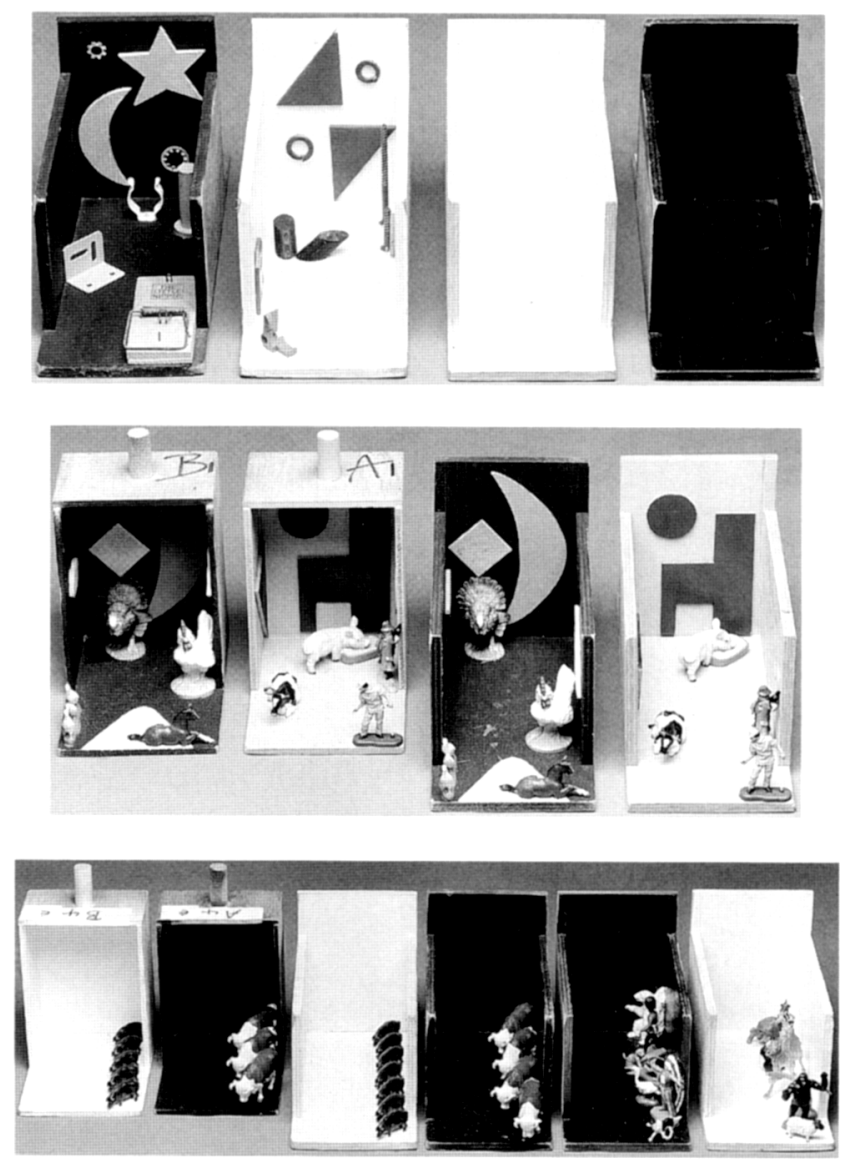

Figure 1. The box types used in Experiments 2 and 3. Top: large black and white empty boxes and identical boxes that contained objects; Middle: large-object boxes matched to smaller boxes; Bottom: large and small boxes containing series of objects, both matched (containing identical objects) and different. 
Table 1

Box-Type Abbreviations, Conditions of Presentation, and Critical Comparisons for Postoperative Delayed Nonmatching to Sample Experiments

\begin{tabular}{|c|c|c|c|}
\hline $\begin{array}{c}\text { Box-type } \\
\text { abbreviation }\end{array}$ & $\begin{array}{l}\text { Presentation } \\
\text { condition }\end{array}$ & Experiment & $\begin{array}{l}\text { Critical box } \\
\text { comparisons }\end{array}$ \\
\hline $\begin{array}{l}\text { SMALL-OBJECT } \\
\text { (Small boxes containing several } \\
\text { different objects) }\end{array}$ & $\begin{array}{l}\text { PTU } \\
\text { rpt. }\end{array}$ & $\begin{array}{l}1 \mathrm{~A}, 1 \mathrm{~B} \\
2 \mathrm{~A}\end{array}$ & \\
\hline $\begin{array}{l}\text { LARGE-B/W-EMPTY } \\
\text { (Large black or white empty } \\
\text { boxes) }\end{array}$ & rpt. & $\begin{array}{l}2 B \\
2 C \\
3 \\
4\end{array}$ & $\begin{array}{l}\text { LARGE-OBJECT (rpt.) } \\
\text { LARGE-SERIES (rpt.) } \\
\text { LARGE-OBJECT (PTU) }\end{array}$ \\
\hline $\begin{array}{l}\text { SMALL-OBJECT-MATCH } \\
\text { (Small boxes containing several } \\
\text { distinctive objects, matched } \\
\text { to LARGE-OBJECT-MATCH } \\
\text { boxes) }\end{array}$ & rpt. & $2 \mathrm{C}$ & LARGE-OBJECT-MATCH (rpt). \\
\hline $\begin{array}{l}\text { LARGE-OBJECT-MATCH } \\
\text { (Large boxes containing several } \\
\text { distinctive obiects matched }\end{array}$ & rpt. & $2 \mathrm{C}$ & SMALL-OBJECT-MATCH (rpt.) \\
\hline $\begin{array}{l}\text { to SMALL-OBJECT-MATCH } \\
\text { boxes) }\end{array}$ & PTU & 4 & $\begin{array}{l}\text { LARGE-B/ w-ERIII II (IPL.) } \\
\text { LARGE (rpt.) }\end{array}$ \\
\hline $\begin{array}{l}\text { LARGE-OBJECT-DIFFERENT } \\
\text { (Large boxes containing several } \\
\text { objects, different from }\end{array}$ & rpt. & $2 \mathrm{C}$ & $\begin{array}{l}\text { LARGE-OBJECT-MATCH (rpt.) } \\
\text { LARGE-B/W-EMPTY (rpt.) }\end{array}$ \\
\hline $\begin{array}{l}\text { objects used in MATCH } \\
\text { boxes). }\end{array}$ & PTU & 4 & LARGE (rpt.) \\
\hline $\begin{array}{l}\text { SMALL-SERIES-MATCH } \\
\text { (Small boxes containing line of } \\
\text { identical objects, matched } \\
\text { to LARGE-SERIES-MATCH } \\
\text { boxes) }\end{array}$ & rpt. & 3 & LARGE-SERIES-MATCH (rpt.) \\
\hline \multirow{2}{*}{$\begin{array}{l}\text { LARGE-SERIES-MATCH } \\
\text { (Large boxes containing line of } \\
\text { identical objects, matched } \\
\text { to SMALL-SERIES-MATCH } \\
\text { boxes) }\end{array}$} & rpt. & 3 & \multirow{2}{*}{$\begin{array}{l}\text { SMALL-SERIES-MATCH (rpt.) } \\
\text { LARGE-B/w-EMPTY (rpt.) } \\
\text { LARGE (rpt.) }\end{array}$} \\
\hline & PTU & 4 & \\
\hline \multirow[t]{2}{*}{$\begin{array}{l}\text { LARGE-SERIES-DIFFERENT } \\
\text { (Large boxes containing line of } \\
\text { different objects) }\end{array}$} & rpt. & 3 & \multirow{2}{*}{$\begin{array}{l}\text { LARGE-SERIES-MATCH (rpt.) } \\
\text { LARGE-B/w-EMPTY (rpt.) } \\
\text { LARGE (rpt.) }\end{array}$} \\
\hline & PTU & 4 & \\
\hline
\end{tabular}

Note. Further details of the boxes are given in the Stimuli section. PTU = pseudotrial unique stimulus presentations; no goal box was presented more than once on any 1 day. Repeated (rpt.) $=$ two goal boxes (only) were presented on any 1 day.

2B-4). They were constructed of plywood and of the following dimensions: $25.4 \mathrm{~cm}$ long and $12.0 \mathrm{~cm}$ wide, with side walls 12.0 $\mathrm{cm}$ high and the rear wall $16.0 \mathrm{~cm}$ high (the end toward the start area was open). LARGE-B/W-EMPTY boxes differed only in paint finish, one being matte white and the other black in each pair. The LARGE-B/W-EMPTY boxes provide a standard comparison condition throughout the study and were therefore most suitable for the preoperative training procedures.

\section{Preoperative Training}

Phase 1: Shaping. The rats were handled over 2 weeks to reduce reactivity and introduced to reward pellets in the home cage over 2 days as food deprivation was introduced. During the third week, rats were placed in pairs (cagemates) in the two-choice apparatus (with the ends blocked off with blank goal boxes) and were encouraged to explore by placing reward pellets throughout the maze. On successive days, the number of pellets was decreased and their location was gradually restricted to the goal boxes. When the rats moved freely in pairs, they were given solo exposure to the maze. The metal guillotine doors were occasionally lowered so that the rats would habituate to the noise and feeling of the door on their tail. This procedure continued until all rats ate freely without defecation in the maze for a total 6 days with up to $1 \mathrm{hr}$ exposure per rat per day.

Phase 2: Repeated LARGE-B/W-EMPTY boxes. After the rats were familiarized with the apparatus, they were trained on the DNMS rule. A pair of LARGE-B/W-EMPTY boxes was used in a discrete trial procedure in a short preoperative phase intended to accustom the rats to the maze and the large goal-box stimuli.

For testing, 5-6 rats were removed from their home cages and were placed in separate holding cages in the testing room. Each rat received only one trial at a time. Thus, the first rat would receive its second trial only after the other 6 rats had received their first (discrete trial procedure). There were four squads of 5-6 rats, each given eight trials per day.

A trial consisted of a forced (information) run and a choice (recognition) run. The rat was placed in the start area for $5 \mathrm{~s}$, after which the door was slowly raised over $1 \mathrm{~s}$ and the rat could enter one of the goal areas (the door to the other being closed). After the 
rat had entered, the guillotine door to that area was lowered. The rat was allowed to explore the goal box until it found at least one of the two reward pellets that had been dropped through the hole above the goal-box area onto the goal-box base (occasionally pellets were inaccessible). The typical goal-box confinement time was 5-7 s to find both pellets. The rat was then returned by hand to the start area. The intratrial interval (time between completion of forced run and beginning of choice run) was $3-5 \mathrm{~s}$. This delay reflects the time required to move the rat from one compartment of the maze to another and to alter box positions if necessary or to remove and replace the boxes when the box positions were to be held constant. The intertrial interval was an estimated average of 6-7 min.

On the choice run, all doors were raised and the rat was allowed to choose between the two goal boxes. The choice criterion was that the rat should place at least three paws simultaneously on the goal box; the door to that goal area was then lowered. If the rat selected the box that was different from the one entered on the forced run (DNMS), it was rewarded with two pellets, delivered as above. The rat was returned to its holding cage, and the next rat began its trial. If the incorrect box was selected, a within-trial correction procedure was used on the first four trials to help to avoid the development of side preferences. The door was not closed after an incorrect choice, and the rat was allowed to enter the other arm and obtain a reward. On the last four trials of the day, an incorrect choice simply led to nonrewarded confinement in the goal box of 5-7 s. The correction procedure was used during preoperative training and in Experiments 1B, 2A, and 2B (in each case for the first half of each day's testing) but not thereafter.

The design was fully counterbalanced throughout. The left-right positions of forced runs and of correct choice runs were switched pseudorandomly across trials. The left-right goal-box positions were altered between the forced and choice runs on half of the trials (also ordered pseudorandomly). Thus the rat learned a cue-relevantspace-irrelevant task. The LARGE-B/W-EMPTY stimui were designated correct $(S+)$ or incorrect $(S-)$ in a balanced semirandom sequence, with the constraint that the same box was not $S+/ S-$ for more than three consecutive trials.

The rats were tested in the same order, 5 days per week, at approximately the same time every day between 9 a.m. and 6 p.m. Each rat received the same sequence of left-right goal-box positioning. The first eight introductory trials were given over 2 days, so that animals reluctant to run could be identified and given additional exposure to reward pellets on the maze. In fact, none of the rats required further exposure to the maze at this point, so the average score of those eight trials is included in the analysis. There were a further 9 days of testing (i.e., a total of 80 trials) in this phase.

Although this was insufficient training for the rats to learn the DNMS task, the mean scores from this phase were used to match maze performance levels between groups before surgery. No rats were excluded at this stage.

\section{Postoperative Testing}

Twenty-two rats were tested postoperatively (see Subjects section above). All testing was conducted with the experimenter uninformed as to the rats' allocation to the experimental groups.

The rats were handled during the postoperative recovery period to reduce general reactivity and then systematically exposed (as before, see Preoperative Training section) to the $Y$-maze (see Apparatus section). They were trained on DNMS using the $Y$-maze task in Experiment 1. In Experiment 2, they were transferred back to the two-choice maze used in preoperative training. In all subsequent experiments, rats were tested on the two-choice maze (see Table 2).

\section{Statistics}

Analyses were carried out using the GENSTAT IV package implemented on a VAX computer. The dependent variable was percentage correct, and data were analyzed in blocks of days (factor blocks). In Experiment 1, a block consisted of 4 consecutive days testing. In Experiments 2 and 3, blocks refer to 4 nonconsecutive days testing under the same box-type condition. In Experiment 4 , a block refers to 2 nonconsecutive days testing under the same box-type condition. Significant interactions were explored with post hoc comparisons made by $t$ test (two-tailed), using the standard error of the difference of the mean (SED) derived from the corresponding stratum of the analysis of variance (ANOVA). All statistical tests use an alpha of .05 .

\section{Experiment 1}

This experiment assessed the effects of hippocampal lesion on postoperative acquisition of the DNMS task in the Y-maze.

Animals can perform successfully on matching tasks without applying a true matching rule. For example, pigeons may learn to choose accurately in a DMS task without being able to apply the response rule to familiar stimuli in an unfamiliar configuration. This suggests that the birds were not matching the stimuli, but had simply learned a number of different conditional responses (Kamil \& Sacks, 1972). Similar conclusions have been drawn from work with rats and monkeys (Iversen, 1993; Sidman, 1992). This kind of stratagem would lead to responding at chance levels if novel stimuli were introduced. We therefore trained the rats in our DNMS study using novel small object boxes in a PTU design to ensure that the rats would acquire a generalizable DNMS rule, readily transferable to the novel boxes used in later experiments. Thus, any disruption of performance caused by novelty as such would be minimized, making any disruption associated with the form of the stimuli easier to identify in subsequent experiments.

In Phase A, rats were trained on the $\mathrm{Y}$-maze. Although this maze is different from the two-choice maze required for the main series of experiments, it allows the use of a continuous training procedure (described below), which is faster than the procedure used in the two-choice maze (described above). We already know that trained rats will transfer learning of matching rules from one maze to the other (Rawlins et al., 1993). In Phase B, they were tested with small object boxes, still on a PTU schedule but now in the two-choice maze and with the discrete trial procedure to be used in the subsequent experiments with large goal-box stimuli.

\section{Method}

\section{Stimuli}

Small object (SMALL-OBJECT) boxes. A set of 22 identical pairs of goal boxes were modeled after those used by Aggleton (1985) for use in Experiments 1A and 1B. Each pair of object boxes could differ in texture, colored paint design, and the objects they contained. The goal boxes were constructed of hardboard, with a base $17.3 \mathrm{~cm}$ long, side walls $8.5 \mathrm{~cm}$ long and $15.8 \mathrm{~cm}$ high, and a 
Table 2

Pre-and Postoperative Training and Testing on Delayed Nonmatching to Sample (DNMS) Rule: Procedural Sequence and Experimental Objectives

\begin{tabular}{|c|c|c|c|}
\hline $\begin{array}{l}\text { Training and } \\
\text { testing }\end{array}$ & Apparatus & Stimuli & Objective \\
\hline Preoperative & $\begin{array}{c}\text { Two-choice } \\
\text { maze. }\end{array}$ & $\begin{array}{l}\text { Large empty boxes, presented } \\
\text { repeatedly. }\end{array}$ & $\begin{array}{l}\text { Introduction to procedures } \\
\text { and large stimuli: perfor- } \\
\text { mance matched for allo- } \\
\text { cation to surgical } \\
\text { groups. }\end{array}$ \\
\hline \multicolumn{4}{|l|}{ Postoperative } \\
\hline Experiment 1A & Y-maze. & $\begin{array}{l}22 \text { identical pairs of small } \\
\text { goal boxes containing } \\
\text { objects, presented } \\
\text { without repetition within } \\
\text { the day's testing. }\end{array}$ & $\begin{array}{l}\text { Rapid training on the DNMS } \\
\text { rule. }\end{array}$ \\
\hline Experiment $1 \mathrm{~B}$ & $\begin{array}{c}\text { Two-choice } \\
\text { maze. }\end{array}$ & $\begin{array}{l}20 \text { different small goal boxes } \\
\text { containing objects, pre- } \\
\text { sented without repetition } \\
\text { within the day's testing. }\end{array}$ & $\begin{array}{l}\text { Transfer of the DNMS rule } \\
\text { (using familiar goal-box } \\
\text { type and schedule) to } \\
\text { apparatus that accom- } \\
\text { modates large goal } \\
\text { boxes and discrete trial } \\
\text { procedures. }\end{array}$ \\
\hline Experiment $2 \mathrm{~A}$ & $\begin{array}{c}\text { Two-choice } \\
\text { maze. }\end{array}$ & $\begin{array}{l}\text { Single pair of small goal } \\
\text { boxes containing } \\
\text { objects, presented } \\
\text { repeatedly. }\end{array}$ & $\begin{array}{l}\text { Transfer of the DNMS rule } \\
\text { (using familiar goal-box } \\
\text { type) to repeated presen- } \\
\text { tation schedule. }\end{array}$ \\
\hline Experiment 2B & $\begin{array}{c}\text { Two-choice } \\
\text { maze. }\end{array}$ & $\begin{array}{l}\text { Single pair of large empty } \\
\text { goal boxes, presented } \\
\text { repeatedly. }\end{array}$ & $\begin{array}{l}\text { Transfer of the DNMS rule to } \\
\text { repeated presentation of } \\
\text { large boxes. }\end{array}$ \\
\hline Experiment $2 \mathrm{C}$ & $\begin{array}{c}\text { Two-choice } \\
\text { maze. }\end{array}$ & $\begin{array}{l}\text { Pairs of large and small goal } \\
\text { boxes containing } \\
\text { matched configurations } \\
\text { of objects; a pair of } \\
\text { large goal boxes con- } \\
\text { taining a different con- } \\
\text { figuration of objects; } \\
\text { and a pair of large } \\
\text { empty goal boxes. All } \\
\text { stimuli were presented } \\
\text { repeatedly. }\end{array}$ & $\begin{array}{l}\text { Determination of DNMS } \\
\text { accuracy for different } \\
\text { goal-box types. } \\
\text { Can hippocampectomized } \\
\text { rats respond on the basis of } \\
\text { (configurations of) objects } \\
\text { within the large boxes? }\end{array}$ \\
\hline Experiment 3 & $\begin{array}{c}\text { Two-choice } \\
\text { maze. }\end{array}$ & $\begin{array}{l}\text { Pairs of large and small goal } \\
\text { boxes containing } \\
\text { matched series of } \\
\text { objects; a pair of large } \\
\text { goal boxes containing a } \\
\text { different series of } \\
\text { objects; and a pair of } \\
\text { large empty goal boxes. } \\
\text { All stimuli were pre- } \\
\text { sented repeatedly. }\end{array}$ & $\begin{array}{l}\text { Determination of DNMS } \\
\text { accuracy for different } \\
\text { goal-box types. } \\
\text { Can hippocampectomized } \\
\text { rats respond on the basis } \\
\text { of series of objects within } \\
\text { the large boxes? }\end{array}$ \\
\hline Experiment 4 & $\begin{array}{c}\text { Two-choice } \\
\text { maze. }\end{array}$ & $\begin{array}{l}20 \text { different large goal boxes } \\
\text { containing objects, pre- } \\
\text { sented without any rep- } \\
\text { etition within each day's } \\
\text { testing; a pair of large } \\
\text { empty goal boxes pre- } \\
\text { sented repeatedly. }\end{array}$ & $\begin{array}{l}\text { Determination of DNMS } \\
\text { accuracy for large goal } \\
\text { boxes presented on a } \\
\text { "trial-unique" schedule, } \\
\text { compared with that for } \\
\text { repeated large empty } \\
\text { goal boxes as a con- } \\
\text { tinuing control for prac- } \\
\text { tice effects. } \\
\text { Can hippocampectomized } \\
\text { rats remember the large } \\
\text { boxes given more cues } \\
\text { and less repetition? }\end{array}$ \\
\hline
\end{tabular}


back wall $11.7 \mathrm{~cm}$ wide and $15.8 \mathrm{~cm}$ high. A top covered the area enclosed by the side walls. Further variants of the SMALL-OBJECT boxes were designed for use in subsequent experiments: These are described under the relevant Method sections.

\section{Procedure}

Experiment 1A. Rats were first accustomed to the $Y$-maze, the noise of the lid and doors, and confinement in the holding cages, as was done previously. With 2 days exposure, first in pairs, second singly, they explored freely and ate the reward pellets without defecation. DNMS testing in the novel apparatus was introduced over 2 days of four trials each, followed by 2 days of six trials each because the rats ran slowly at first. Thereafter, they were trained at 10 trials per day on the DNMS rule. Over the first 5 days on the $Y$-maze, a number of rats required breaks in the testing before they would run. These data were not included in the analysis. There were a further 40 days of training, as described below.

Eleven pairs of SMALL-OBJECT boxes were used each day, with the constraint that no goal-box stimulus was repeated on consecutive days (there were 2 pools of 11 pairs of boxes in use on alternate days). Members of a pair were identical to each other. When performing DNMS, the rats were given a choice between a (relatively) novel goal box and one that matched the sample in decoration and the objects it contained. The task was to select the relatively novel goal box. All rats received the same randomized goal-box order and left-right positioning on a given day.

Rats were given 10 trials per day in a continuous procedure; each rat received all of its daily trials before the next rat was tested in sequence. The rats were tested in batches of 5-6, as was done previously. Correction procedures are an integral part of the routine in the $Y$-maze because in the continuous testing procedure, the last stimulus selected provides the sample for the next trial. On any "single" trial, the rat was given four choices between the rewarded sample and a second stimulus until it selected the relatively novel second goal box or was forced there on the fifth occasion and rewarded as usual. During these within-trial corrections, the right-left positions of boxes were swapped so that the correct selection required the rat to make the right-left body turn originally designated for that trial. The rat then ended up in the rewarded sample for the next trial to begin. This procedure helps to avoid the development of side preferences.

Experiment $1 B$. Twenty different SMALL-OBJECT boxes were used each day in the two-choice maze. The first 10 trials were run over 2 days because the rats were relatively unaccustomed to performing the task in the two-choice maze (the apparatus was familiar from preoperative training). The rats made their choices relatively slowly on the first 2 days, but choice accuracy was comparable to that at the end of training on the $Y$-maze. The data from the first 2 days were therefore included in the analysis and a further 7 days was run, to a total of 80 trials. Testing procedures were otherwise as described for preoperative training. Correction procedures were applied in this phase.

\section{Results}

\section{Histological}

All 6 CORT rats had sustained bilateral neocortical damage that had sectioned the corpus callosum, exposing the alveus of the dorsal hippocampus without resulting in any direct damage to the hippocampus. The damage was bilateral at the anterior plane of assessment in all 6 rats (see Figure 2A) and was bilateral at the intermediate plane in 3 of them; the remaining 3 had no damage at this plane. One rat had restricted unilateral cortical damage at the posterior plane. Despite the lack of hippocampal damage, three rats in the CORT group showed patches of gliosis in the underlying thalamus, which was bilateral in two of them.

Two of the HIPP rats showed evidence of postoperative infection that had caused extensive damage outside the intended target structures. Both were excluded from further analysis. The remaining 7 HIPP rats were retained in the study. All had sustained extensive bilateral hippocampal damage at the anterior plane of assessment, with a complete ablation in 6 of them and sparing restricted to the most medial parts of the structure in the 7th. Cortical damage at this plane was comparable to that observed in the CORT group. There was also extensive bilateral damage at the intermediate plane, with a complete ablation in 4 subjects, moderate unilateral sparing in 1, and slight bilateral sparing in 2 subjects. One subject had restricted bilateral cortical damage at this plane, and 1 had very restricted unilateral damage; the neocortex was undamaged in the remainder. There was clear bilateral damage in 5 subjects at the posterior plane of assessment, and extensive unilateral damage in the 2 others; none of the subjects had neocortical damage. One rat showed a unilateral near-complete section of the cingulum bundle; there was no other damage to this structure. Two rats had restricted unilateral damage to the entorhinal cortex; there was no entorhinal damage in the remainder. All 7 rats showed discrete bilateral patches of gliosis below the surface of the thalamus; in 5 rats there was also bilateral roughening of the surface of the thalamus, though no visible penetration damage; in the remaining 2 rats there was unilateral roughening. Thalamic damage of this kind has been observed before in rats with hippocampal aspiration lesions (e.g., Rawlins \& Tsaltas, 1983), and none of these subjects were excluded from the study. Figure 2 (B, $\mathrm{C}$, and D) shows sections of the HIPP rat with an intermediate size of hippocampal lesion and the median extent of thalamic damage (at the anterior, intermediate, and posterior planes of assessment).

\section{Behavioral}

Experiment 1A. There was a main effect of Blocks, $F(9$, $153)=22.66$. The Blocks $\times$ Lesion interaction was also significant, $F(18,153)=2.30$. Figure 3 shows that although the sham group tended initially to perform worse than the lesioned groups, near the end of training this pattern was temporarily reversed. Post hoc $t$ tests confirm that the shams were significantly more accurate than the HIPPs on Blocks 8 and $9, t s(153)=2.14$ and 3.21, respectively. By Block 10 , the tendency for worse performance in the HIPPs was preserved, but the control groups did not differ significantly from each other. No other differences were significant.

Experiment $1 B$. DNMS performance on the PTU schedule with small boxes transferred to the two-choice maze with an overall mean of $79 \%$ for the 80 trials of testing. ANOVAs showed a main effect of Days, $F(7,119)=4.66$, but there were no significant effects involving Lesion. 


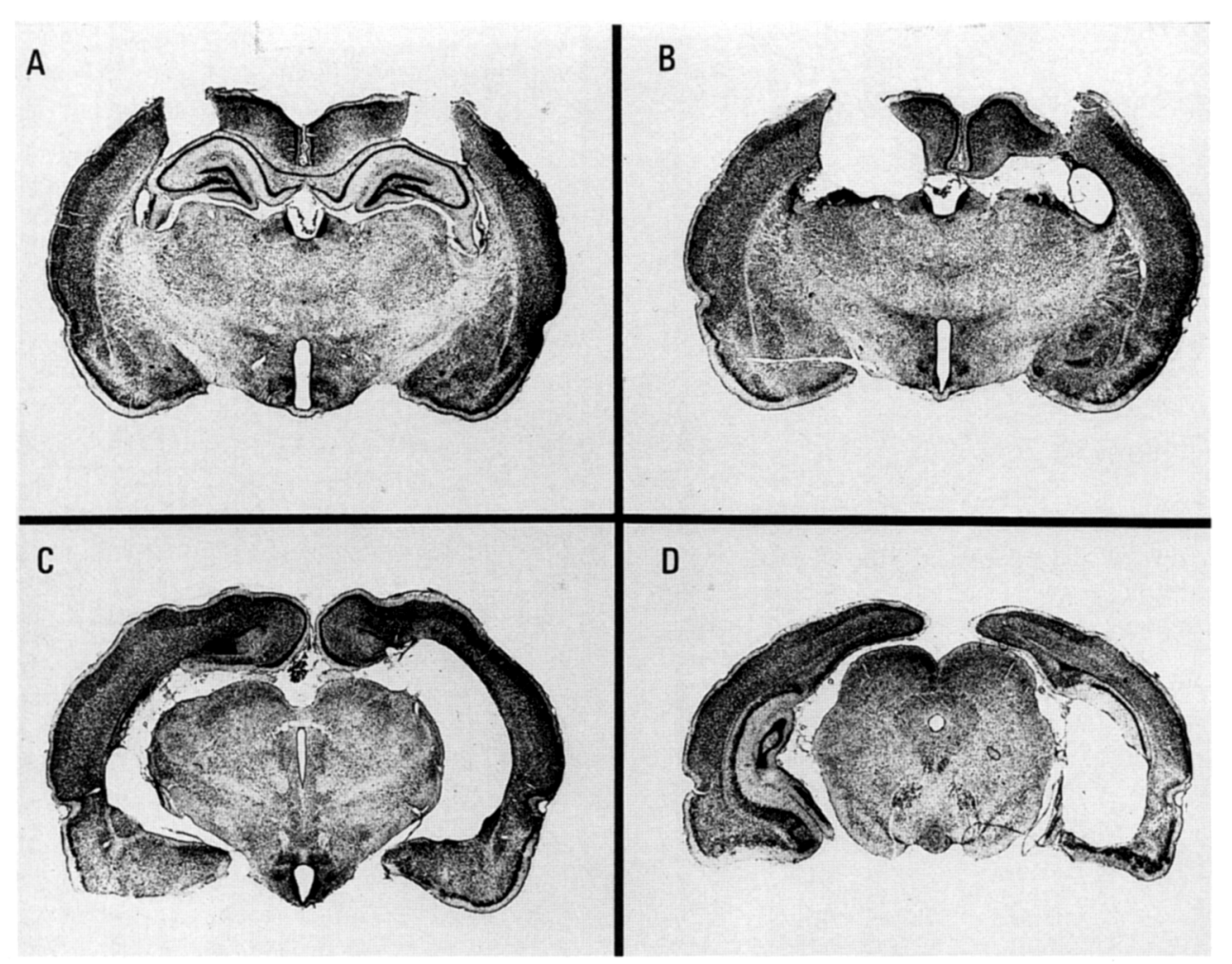

Figure 2. Coronal sections of rat brain, stained with cresyl violet, at the three planes of assessment approximately corresponding to Plates 32, 38, and 43 in the atlas of Paxinos and Watson (1986), showing the following: (A) The lesion in the rat from the cortically lesioned group with the median lesion size at the anterior plane of assessment (there was no damage at intermediate or posterior planes of assessment); (B, C, and D) The lesion in the rat from the hippocampally lesioned group with the median degree of thalamic damage and a median hippocampal lesion at the anterior, intermediate, and posterior planes of assessment, respectively.

\section{Discussion}

After 40 days of training in Experiment $1 \mathrm{~A}$, a stable baseline of performance on DNMS was reached. This was still relatively low but adequate for our purposes because the subsequent experiments provided further training on the DNMS rule. The use of within-groups comparisons to determine the effects of box type allowed control for shifts in the baseline level of performance.

In Experiment 1B, the rats transferred performance of this . task to the two-choice maze in which subsequent testing took place.

We have previously reported that hippocampally lesioned rats show no acquisition deficit when trained postoperatively using SMALL-OBJECT stimuli presented pseudo-trial-uniquely (Rawlins et al., 1993). In the present experiment, again no enduring lesion-induced deficit was observed.

\section{Experiment 2}

We next assessed the effects of hippocampal lesions on DNMS using repeated presentation of goal-box stimuli in the two-choice maze, designed to accommodate the large goal-box stimuli. This allowed us directly to compare performance with large and small boxes. Experiment 2 wás divided into three phases (A, B, and $\mathrm{C}$ ). Because we planned to compare performance with large and small object goal boxes, Experiment $2 \mathrm{~A}$ introduced repetition of SMALLOBJECT boxes, familiar from Experiment 1. Experiment 2B then introduced repetition of the LARGE-B/w-EMPTY boxes, familiar from preoperative training. In Experiment $2 \mathrm{C}$, we compared performance with a variety of box types, tested in a rotating sequence.

\section{Method}

\section{Stimuli}

SMALL-OBJECT boxes. A single pair of the SMALL-OBJECT goal boxes used in Experiment 1 was used in every trial in Experiment $2 \mathrm{~A}$. In Experiment $2 \mathrm{C}$, new small object boxes were constructed, matching large object boxes in every respect except size and details of construction (SMALL-OBJECT-MATCH, see Figure 1 [middle]).

LARGE-B/W-EMPTY. These were the large empty boxes described for preoperative training (see Figure 1 [top], right pair).

Large object (LARGE-OBJECT) boxes. Boxes of identical size and construction to the LARGE-B/W-EMPTYS contained arrays of objects and decoration. In Experiment 2C, one pair of LARGE-OBJECTMATCH boxes (see Figure 1 [middle], right pair) matched the SMALL-OBJECT-MATCH boxes in use; another LARGE-OBJECT-DIFF pair 


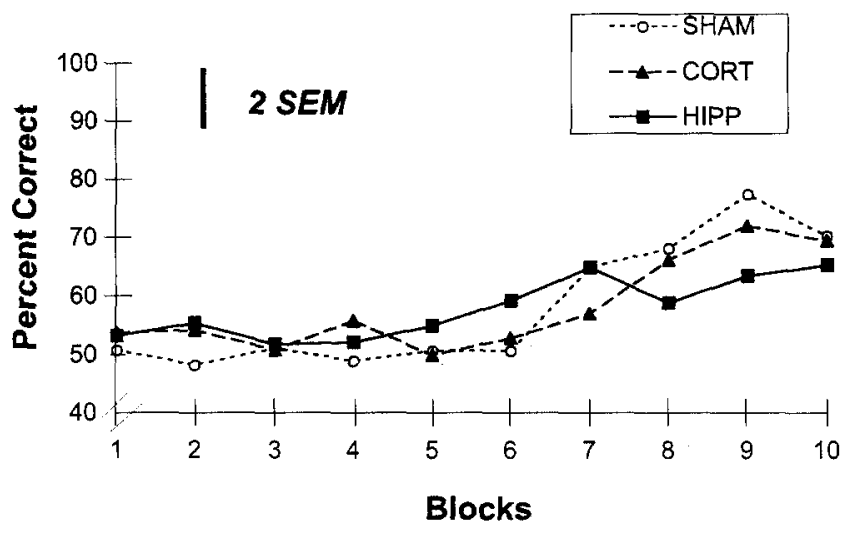

Figure 3. Line graph shows acquisition of delayed nonmatching to sample (as mean percent correct), using trial-unique presentation of small object boxes. Blocks refer to averages for every 4 days of testing. Bar at top left shows two standard errors of the difference of the mean $(S E M)$ for between-groups comparisons for the significant Lesion $\times$ Blocks interaction, taken from the appropriate stratum of the analysis of variance. SHAM = sham-operated controls; CORT = cortically lesioned group; HIPP = hippocampally lesioned group.

had different decoration and contained a different set of objects (see Figure 1 [top], left pair)

\section{Procedure}

Experiment 2A: SMALL-OBJECT boxes. The rats were tested on DNMS for 4 days using a pair of SMALL-OBJECT boxes presented repeatedly in the two-choice maze. Rats were run in four squads of 5-6 rats in a discrete trial procedure. All rats received the same randomized goal-box order and left-right positioning on a given day. Testing methods were otherwise identical to those described in the preoperative training section, with correction procedures on the first five trials of each day.

Experiment 2B: $L A R G E-B / W-E M P T Y$ boxes. The rats were tested on DNMS for 4 days using a pair of LARGE-EMPTY boxes presented repeatedly in the two-choice maze, as above.

Experiment 2C: LARGE-OBJECT boxes. In this, the main phase, four experimental conditions were run in a 4-day rotation in the two-choice maze. DNMS was tested using four pairs of goal boxes: (a) SMALL-OBJECT-MATCH; (b) LARGE-OBJECT-MATCH (these goalboxes differed only in box size, the objects they contained and decoration were identical); (c) LARGE-OBJECT-DIFF, different to the above; (d) LARGE-B/W-EMPTY. The sequence in which the conditions was run was unpredictable, with the constraint that the same condition was not run on consecutive days and the sequence was different for each block of testing. There was a total of five 4-day blocks with 10 trials per day. There were thus 50 determinations of performance under each test condition. Basic DNMS procedures were again as described for preoperative training, with the exception that correction procedures were no longer in use.

\section{Results}

\section{Experiment $2 A$ and $2 B$}

We used ANOVAs to compare performance on the two 4-day blocks with repeated SMALL-OBJECT and LARGE-B/wEMPTY boxes. There were significant main effects of lesion,
$F(2,17)=15.46$, and Box-type, $F(1,17)=8.55$, together with a significant Lesion $\times$ Box-Type interaction, $F(2,17)=$ 4.79. The change in testing conditions produced a fall in accuracy within subjects, significant in the HIPP group, $t(17)=3.72$, but short of significance in the CORT group, $t(17)=1.97$.

\section{Experiment $2 C$}

There were main effects of Lesion, $F(2,17)=23.67$, and Box-Type, $F((3,51)=12.78$. There was also a significant Box-Type $\times$ Lesion interaction, $F(6,51)=7.66$. Blocks was significant as a main effect, $F(4,272)=3.13$, and in interaction with Box-Type, $F(12,272)=2.49$. Neither of the interactions involving lesion and blocks was significant, so Figure 4 shows mean percent correct averaged over days.

Figure 4 shows a high level of performance with the SMALL-OBJECT-MATCH boxes, irrespective of lesion condition. In the LARGE-OBJECT-MATCH, LARGE-OBJECT-DIFF, and LARGE-EMPTY conditions, there was a clear impairment in DNMS performance in the HIPP group only. In all box-type conditions there was a nonsignificant tendency for the CORTs to perform worse than the shams; maximum $t(51)=$ 1.40. Because of the tendency toward impairment in the CORT group, we applied the post hoc tests to the critical CORT and HIPP groups. These comparisons confirmed that the HIPP group was impaired relative to the CORTs with each of the large box types: LARGE-OBJECT-MATCH, $t(51)=$ 3.15; LARGE-OBJECT-DIFF, $t(51)=4.38$; and LARGE-EMPTY, $t(51)=5.32$. The HIPP group was also impaired relative to the shams with each of the large box types; minimum $t(51)=4.61$, but no other between-group comparisons were significant.

Within-groups comparisons confirm that the HIPP group was selectively impaired in the large-box conditions, particularly when the boxes were empty. The transition from small to large OBJECT-MATCH boxes produced a significant fall in accuracy in the HIPP group, $t(51)=5.50$. Although performance with LARGE-OBJECT-DIFF boxes was no different from that with the LARGE-OBJECT-MATCH boxes, the transition from LARGE-OBJECT-DIFF to LARGE-EMPTY boxes produced a further fall off in performance, $t(51)=3.30$. Thus for the HIPP group, the use of any large box resulted in a fall off in performance and LARGE EMPTY boxes made performance worse than in any other condition.

For both the sham and CORT groups, performance with LARGE-EMPTY boxes was worse than that with LARGE-OBJECTDIFF boxes, $t \mathrm{~s}(51)=3.01$ and 2.06 , respectively, but not different from that with SMALL- or LARGE-OBJECT-MATCH boxes. This pattern of effects arises because LARGE-OBJECTDIFF boxes are easier than any other condition for both of the control groups. This is not the case for the HIPP group, for which these boxes are clearly harder to deal with than SMALL-OBJECT-MATCH boxes, $t(51)=4.77$, equivalent to the LARGE-OBJECT-MATCH boxes, and easier than the LARGEEMPTY boxes, $t(51)=3.30$.

\section{Discussion}

In Experiment 2A, the rats transferred performance of the DNMS rule when a single pair of SMALL-OBJECT boxes was 


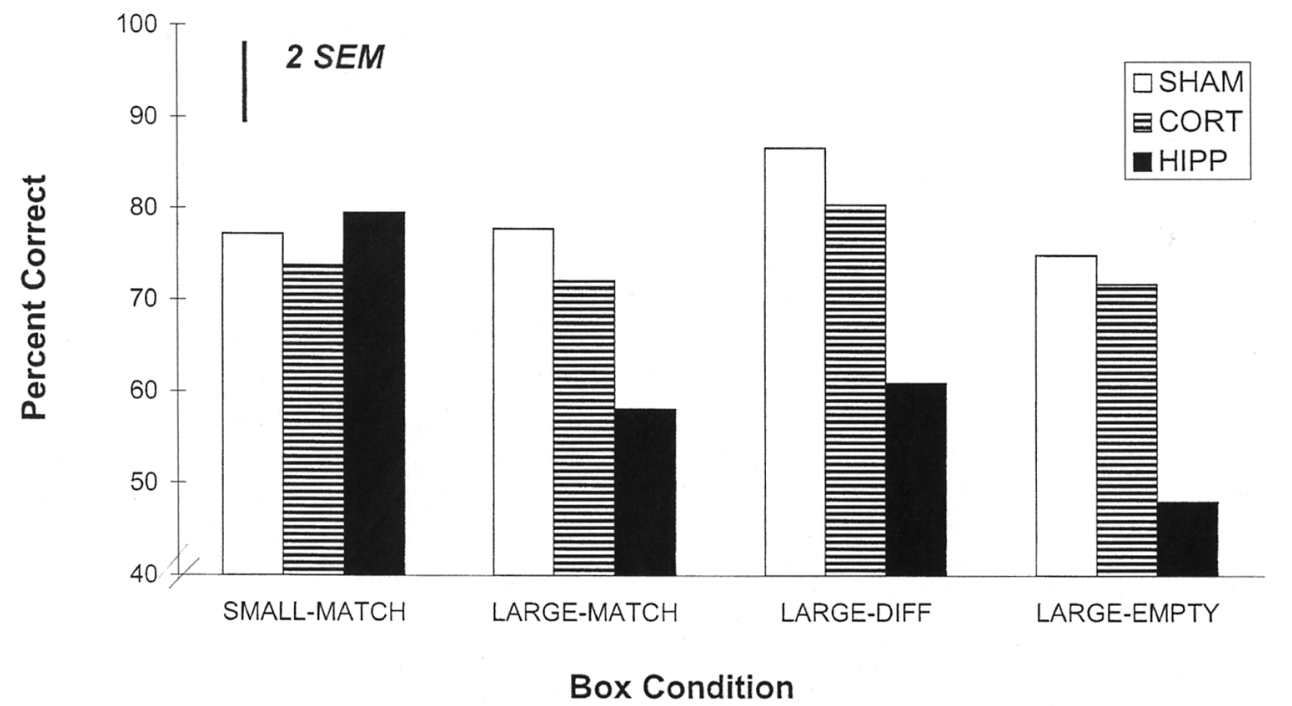

Figure 4. Bar histogram showing delayed nonmatching to sample choice accuracy (mean percent correct) for hippocampally lesioned group (HIPP), cortically lesioned group (CORT), and sham-operated group (SHAM) under four box-type conditions: SMALL-MATCH (small object boxes); LARGE-MATCH (large object boxes decorated to match the SMALL-MATCH boxes); LARGE-DIFF (large object boxes different from the above); LARGE-EMPTY (large black or white empty boxes). Bar at top left shows two standard errors of the difference of the mean $(S E M)$ for between-groups comparisons, for the significant Lesion $\times$ Box-Type interaction, taken from the appropriate stratum of the analysis of variance.

presented repeatedly. In Experiment $2 \mathrm{~B}$, they were reintroduced to the LARGE-B/W-EMPTY boxes. The switch in box types produced a fall off in performance accuracy that was significant only after hippocampectomy. The tendency for the cortical control group to be similarly affected is discussed further below.

In Experiment 2C, the effect of Lesion resulted from a clear drop in DNMS accuracy whenever the HIPP rats were tested with large boxes (Figure 4). This occurred irrespective of the degree of complexity of the large boxes used and whether or not they resembled the SMALL-OBJECT boxes. This was true even when the large boxes contained objects identical to those used in their small box counterparts (SMALL-OBJECT-MATCH), simply spaced further apart (LARGEOBJECT-MATCH). Comparison of performance with LARGEand SMALL-OBJECT-MATCH boxes therefore suggests a strong effect of box size as a determinant of the Lesion effect. This conclusion is supported by the significant Box-Type $\times$ Lesion interaction, as explored above: Post hoc tests confirm that the HIPP group was significantly impaired with each of the large box types. The nonsignificant tendency for the CORTs to perform relatively worse than the shams at this stage of postoperative testing was comparable in all boxtype conditions. The within-groups comparisons further confirm that the transition from small to large boxes produced a significant fall in accuracy selectively in the HIPP group.

The Lesion effects shown in Figure 4 bear no obvious relationship to task difficulty: There was still a clear effect of the HIPP lesion in the LARGE-OBJECT-DIFF condition, easiest for both control groups.

\section{Experiment 3}

Since the HIPP rats were still impaired with the above LARGE-OBJECT boxes, we next explored the possibility that a continuous chain of objects from the choice point to the point of reinforcement might make the DNMS task soluble for the lesioned rats. Such a series was predicted to help the rats to bridge the associative distance from the choice point to the point of reinforcement (Cassaday \& Rawlins, 1995; Leaton \& Borczcz, 1990). We also tested to see whether this distance might be more effectively bridged by identical objects repeating back from the choice end of the box to the end where reward would be delivered or by a series of nonidentical objects. Both series provided the rat with an object at the choice point and at the point of reinforcement, and each contained roughly the same density of objects. Any difference in performance between the conditions would therefore inform an account on the basis of associative distance. We predicted that a series of identical objects would be easiest because in this condition there would be no associative distance to bridge (a sequence of identical items requires the rat only to respond appropriately to an exemplar; e.g., "remember the pig").

The differences in decoration between the LARGE-OBJECTMATCH and LARGE-OBJECT-DIFF boxes seemed unimportant, so we inserted our continuous lines of objects in large plain black or white boxes. These were the identical objects (LARGE-SERIES-MATCH) or distinctively different ones (LARGESERIES-DIFF). In a comparison condition, we tested the rats with SMALL-SERIES-MATCH boxes, identical in all but size and box construction to LARGE-SERIES-MATCH boxes: The smaller 
boxes necessarily contained fewer objects, but the spacing of the objects was equivalent to that in the larger boxes. The rats were again tested with plain LARGE-B/W-EMPTY boxes (that did not contain objects) in this phase.

\section{Method}

\section{Stimuli}

LARGE-EMPTY boxes. A fresh pair was used in Experiment 3 to control for newness when further novel goal boxes were introduced.

Large object series (LARGE-SERIES) boxes. Boxes identical in size, construction, and decoration to the LARGE-B/W-EMPTY boxes contained continuous lines of objects down one side of the box. LARGE-SERIES-MATCH boxes contained a continuous series of repeating identical objects (see Figure 1 [bottom], center pair). LARGESERIES-DIFF boxes contained a continuous line of distinctively different objects (see Figure 1 [bottom], right pair). The LARGESERIES boxes were also used in Experiment 4.

Small object series (SMALL-SERIES-MATCH) boxes. These were small object boxes (see Figure 1 [bottom], left pair) containing continuous lines of objects down one side of the box and matching the LARGE-SERIES-MATCH boxes in every respect, except size and details of construction (as described previously for conventional SMALL-OBJECT boxes).

\section{Procedure}

The rats were tested on DNMS in the two-choice maze under four experimental conditions, using SMALL-SERIES-MATCH, LARGESERIES-MATCH, LARGE-SERIES-DIFF and LARGE-B/W-EMPTY goal boxes. These different test conditions were again presented in an unpredict- able semirandom sequence (adjacent blocks did not start and end with the same condition and the sequence was different in each block) for a total of four 4-day blocks with 10 trials per day. There were thus 40 determinations of performance under each test condition. DNMS procedures were as previously described (without any correction procedures).

\section{Results}

There were main effects of Lesion, $F(2,17)=19.53$, and Box-Type, $F(3,51)=6.97$. There was a significant Box $\times$ Lesion interaction, $F(6,51)=6.44$. There was also a significant Box-Type $\times$ Blocks interaction, $F(9,204)=$ 2.59 , and a Lesion $\times$ Blocks interaction, $F(6,204)=2.27$, but the three-way interaction with Blocks was not significant. Figure 5 therefore shows mean percent correct averaged over days.

Figure 5 shows that although the different lesion groups performed very comparably when tested with SMALL-SERIESMATCH boxes, the HIPP group was consistently impaired with the large box types. In Experiment 3, the tendency, first seen in Experiment 2, for the CORT group to show relative impairment with the larger boxes reached statistical significance in the LARGE-B/W-EMPTY and LARGE-SERIES-MATCH conditions, $t \mathrm{~s}(51)=2.67$ and 2.12 , respectively. The critical post hoc comparisons again assess the effects of the hippocampal lesion against the CORT control group. These comparisons confirm that there was a significant impairment in the HIPP group under both the LARGE-SERIES-MATCH condition, $t(51)=3.93$, and the LARGE-B/W-EMPTY condition, $t(51)=4.3$. In both the SMALL-SERIES-MATCH and

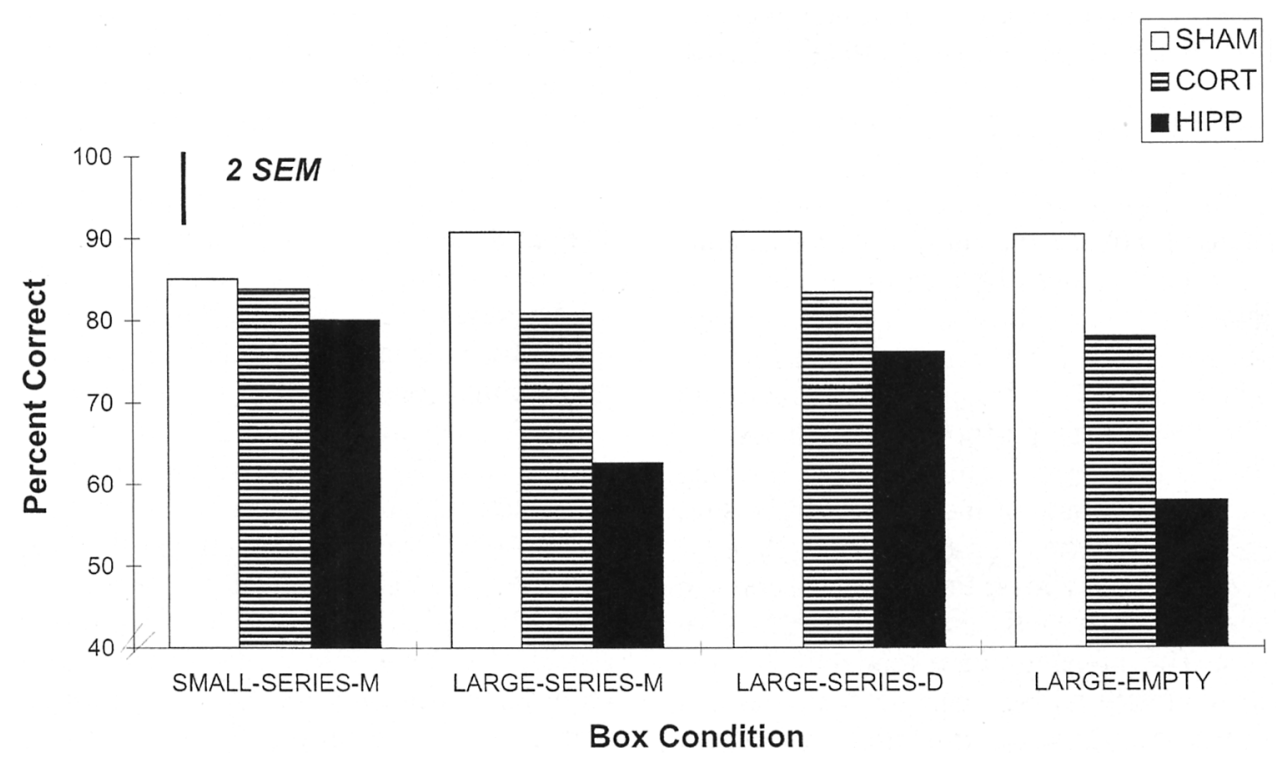

Figure 5. Bar histogram showing delayed nonmatching to sample choice accuracy (mean percentage correct) for hippocampally lesioned group (HIPP), cortically lesioned group (CORT), and sham-operated group (SHAM) under four box-type conditions: SMALL-SERIES-M (small boxes containing a series of identical objects); LARGE-SERIES-M (large boxes containing a matched series of identical objects); LARGE-SERIES-D (large boxes containing a series of distinctively different objects); and LARGE-EMPTY (large black or white empty boxes). Bar at top left shows two standard errors of the difference of the mean (SEM) for between-groups comparisons, for the significant Lesion $\times$ Box-Type interaction, taken from the appropriate stratum of the analysis of variance. 
LARGE-SERIES-DIFF condition, the HIPP group was not significantly impaired relative to the CORT control group. There were no significant differences between the groups with the SMALL-SERIES-MATCH boxes.

Within groups, there were no differences between the boxes within the sham groups; maximum $t(51)=1.60$, and there were no differences between the boxes within the CORT group; maximum $t(51)=1.51$. For the HIPP group (only), performance with LARGE-B/W-EMPTY and LARGESERIES-MATCH boxes was worse than that with both the SMALL-SERIES-MATCH and the LARGE-SERIES-DIFF boxes; minimum $t(51)=3.80$. Thus, the within-subjects comparisons confirm that the HIPP group deficit (otherwise seen whenever the larger boxes are presented) is significantly reduced when these boxes contain a series of distinctive objects.

\section{Discussion}

As in Experiment 2, the lesions had no effect on DNMS performance with the small boxes, but with matched larger boxes, similar in all but size and construction, the lesion effect reappeared. Again within-subjects comparisons confirm that the drop in accuracy typically seen with large goal boxes is selective to the HIPP group. However, this impairment was reduced to a nonsignificant tendency in the condition in which boxes contained a series of distinctively different objects. This suggests that hippocampally lesioned rats might make use of (relative) novelty in performing matching tasks. Objects were not absolutely novel because goal boxes were presented repeatedly, but the number of exposures to the objects in the identical series (used in both small and large boxes) was 7-12 times greater than the number of exposures to each of the nonidentical objects. Consistent with this hypothesis, we have previously observed a tendency for fornix-sectioned rats to show a relative superiority with PTU presentation of small object boxes (e.g., Cassaday \& Rawlins, 1995). We now suggest that a sufficient density of salient objects that are relatively unfamiliar to the rats may significantly alleviate the impairment otherwise seen in the HIPP group.

Differences in general task difficulty do not explain the results because the size of the deficit in the HIPP group bore no obvious relation to the level of performance in control groups (see Figure 5).

\section{Experiment 4}

In the final experiment of the study, we presented LARGE-OBJECT boxes trial uniquely without repetition on any one day. This enabled us to further increase the novelty and distinctiveness of (the objects in) the large goal boxes. ${ }^{1}$ We alternated days of PTU presentation with days of repeated presentation of LARGE-B/W-EMPTY boxes, as previously, so that there was still a baseline measure of any lesion effect. Thus, although we had a smaller set of object boxes, as in Experiment 1, they were not used on consecutive days. Further control for the use of odor cues (using identical pairs of LARGE-OBJECT boxes) was not necessary at this point (see General Discussion section below).

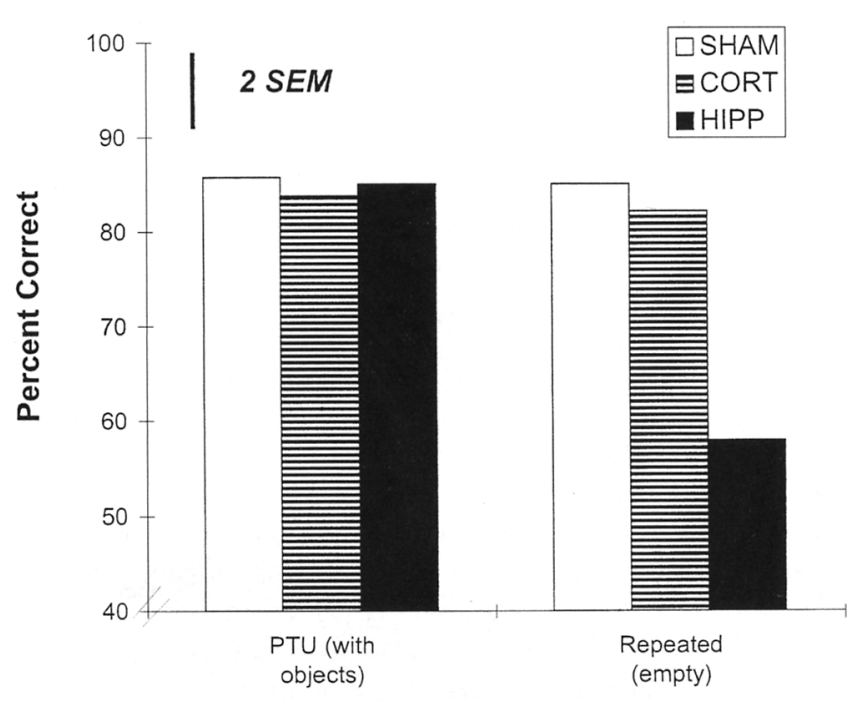

Large Box Condition

Figure 6. Bar histogram showing delayed nonmatching to sample choice accuracy (mean percent correct), using trial-unique presentation of large-object boxes (PTU) and repeated presentation of large black or white empty boxes (repeated). Bar at top left shows two standard errors of the difference of the mean (SEM) for between groups comparisons, for the significant Lesion $\times$ BoxCondition interaction, taken from the appropriate stratum of the analysis of variance. SHAM = sham-operated controls; CORT $=$ cortically lesioned group; HIPP = hippocampally lesioned group.

\section{Method}

A fresh pair of LARGE-B/W-EMPTYS (Figure 1 [top], right pair) was used in Experiment 4 to control for newness when further novel goal boxes were introduced. In this repeated presentation condition, standard DNMS methods were followed (as above). Additional LARGE-OBJECT boxes were devised to allow PTU presentation. We also used all the different large boxes from previous experiments (e.g., Figure 1 [top], left pair; Figure 1 [middle], right pair; Figure 1 [bottom], middle pair and right pair). This provided a pool of 20 boxes for use on any one day. Testing procedures were identical to those used in Experiment 1B. There were 8 days testing in all, at 10 trials per day, giving a total of 40 trials in each experimental condition.

\section{Results}

There were significant main effects of Lesion, $F(2,17)=$ 11.76, and Box-Condition, $F(1,17)=26.50$. There was a significant Box-Condition $\times$ Lesion interaction, $F(2,17)=$ 19.40. Although there was also a significant Box-Condition $\times$ Blocks interaction, $F(3,102)=3.33$, neither of the interactions including Lesion and Blocks was significant. Figure 6 shows a complete recovery of performance in the HIPP group given trial-unique presentations of the larger boxes. This contrasts sharply with the significant drop in performance in the HIPP group with LARGE-B/w-EMPTYS presented repeatedly within the session. A post hoc $t$ test

\footnotetext{
${ }^{1}$ Experiment 4 follows directly from a suggestion made by an anonymous reviewer of Cassaday and Rawlins (1995).
} 
confirmed that this impairment in the HIPP group remained significant relative to the CORTs, $t(17)=6.02$. Further within-subjects comparison confirmed that the drop in performance in the HIPP group with the LARGE-B/W-EMPTYS was significant, $t(17)=8.06$. No other differences were significant.

\section{Discussion}

Rats with hippocampal lesions were unimpaired in DNMS performance with LARGE-OBJECT boxes that were not used repeatedly within a session. At the same time, these same hippocampal rats were still not significantly above chance on DNMS with the LARGE-B/W-EMPTY boxes. Figures 4-6 show this directly because in the LARGE-B/W-EMPTY condition, the hippocampal rats are never more than 2 SEMs above $50 \%$ correct. There is also little change in performance with additional experience: The mean DNMS performance with the LARGE-B/W-EMPTYS ranges between $48 \%$ and $58 \%$ across Experiments $2 \mathrm{C}$ to 4.

Because the hippocampal rats' performance with LARGE-B/ W-EMPTYS remained poor, the recovery in performance with LARGE-OBJECT boxes presented on the PTU schedule cannot be attributed to some general recovery of performance with prolonged testing.

\section{General Discussion}

Rats with hippocampal lesions have substantial and enduring deficits in some nonspatial working memory tasks (Jagielo, Nonneman, Isaac, \& Jackson-Smith, 1990; Olton \& Feustle, 1981; Raffaele \& Olton, 1988) but not in all (Aggleton, Blindt, \& Rawlins, 1989; Aggleton et al., 1986; Mumby, Wood, \& Pinel, 1992; Rothblat \& Kromer, 1991; Shaw \& Aggleton, 1993). We have previously reported that the critical factors determining whether or not these lesions affect object matching or nonmatching to sample are the size complexity, and repetitious use of the stimuli (Cassaday \& Rawlins, 1995; Rawlins et al., 1993).

When the stimuli in use are small objects, retentioninterval duration is another factor that may determine whether hippocampal lesions affect DNMS performance (e.g., Mumby et al., 1992). We did not specifically investigate delay dependence because we continue to observe a robust effect of hippocampal lesions on DNMS with large boxes when testing with the minimum delay required to switch box positions (cf. Raffaele \& Olton, 1988). The fact that deficits in conventional tests of spatial memory are not typically delay dependent either (cf. Kesner, 1991) may be taken to provide further support for the view that (like spatial stimuli) the contextual stimuli provided by our large stimuli engage in encoding system that is vulnerable to disruption by hippocampal damage.

The present experiments further examined the factors of size, complexity, and repetitious use of the stimuli, which we have found to be critical determinants of matching performance in hippocampal rats in order to extend the analysis of the interactions between them and to address an account of the importance of stimulus size, in terms of associative distance. The results confirm previous reports that hippocam- pectomized rats can solve DNMS tasks using small, but not large, goal boxes; this effect was still clear even when the large boxes contained the same objects as the small boxes. However, we went on to demonstrate, for the first time, that this deficit is ameliorated if the large goal boxes contain a sufficient number of differing objects. Moreover, the deficit was eliminated when large boxes were used in a pseudo-trialunique manner, rather than repeated within a session.

We have previously proposed (Cassaday \& Rawlins, 1995; Rawlins et al., 1993) that hippocampal damage leaves rats able to respond flexibly in DMS or DNMS when the stimuli are objects, but unable to do so when the stimuli are contexts. When such tasks use repeated stimulus presentations, the stimulus-response relations required have to be flexible. The rat must switch between selecting and avoiding the same stimulus, depending on whether or not it was arbitrarily designated as the sample on a given trial. Rats with hippocampal dysfunction cannot solve such tasks with large empty goal boxes (Cassaday \& Rawlins, 1995; Rawlins et al., 1993). This contrasts with discrimination learning, in which the correct response to a given stimulus is always the same: Hippocampectomized rats then show no deficit with large, empty goal boxes (Rawlins et al., 1993). Nor does hippocampectomy necessarily impair DMS or DNMS performance using repeated presentations of small object boxes (Cassaday \& Rawlins, 1995; present experiments); hence flexible responding to contextual stimuli seems selectively impaired. The results of Experiment 4 conformed to this pattern, because the deficit seen when large boxes were used was eliminated when those boxes were used without repetition.

\section{Anatomical Substrates of the Dissociation}

Conventional lesions to the hippocampus not only destroy the hippocampus itself but also disconnect structures normally dependent on associated fibre tracts. Further work is needed to clarify the minimal damage necessary for a deficit with large (simple) goal-box stimuli. Our analysis to date suggests that more restricted lesions that impair matching performance with large boxes will not result in a deficit under all circumstances of testing: It should be generally true that any such impairments will be ameliorated when large boxes are made more distinctive. Follow-up experiments will determine whether the same behavioral dissociations are seen with cytotoxic lesions.

There were some tendencies for the cortical lesion to impair DNMS performance. The nonsignificant trend seen in Experiment 2 when larger goal boxes were in use reached conventional levels of significance in Experiment 3 both with large empty boxes and with large boxes containing a line of identical objects. But this effect was again no more than a (very slight) trend in Experiment 4 . Thus effects of the cortical lesion on DNMS performance with large boxes were not reliable under our conditions of testing. In more visually demanding working memory tasks, the effects of cortical lesion can exceed those of hippocampal lesions (Deacon \& Rawlins, 1995). The possibility that (conventional) cortical lesions may disrupt matching performance deserves further 
investigation, particularly in more visually demanding task variants (e.g., see Gaffan \& Woolmore, 1996).

\section{Fact or Odor Artifact?}

It has recently been suggested that rats can solve some versions of nonspatial DNMS task by using odor cues to discriminate which object was more recently handled by the experimenter, rather than by remembering which object had been presented at the sample stage (Herremans, Hijzen, \& Slangen, 1995). We dismiss the possibility of some artifactual explanation of the present pattern of results; for example, they cannot be accounted for by the use of scents deposited by experimenter or rat. First, the experimenter effect (Herremans et al., 1995) depends upon the use of a nonrandomized order of stimulus presentation, but rats can learn DNMS and DMS tasks when this order is randomized (e.g., Aggleton et al., 1986, 1989; Mumby, 1995; Rawlins et al., 1993). Even if some unintended cue of this kind was available in the present experiments, it could not account for the results we obtained, because the experimenter followed an identical protocol regardless of the kind of box used as a stimulus, and yet the HIPP rats showed a performance deficit with some kinds of boxes and no deficit with others (cf. Cassaday \& Rawlins, 1995). Second, boxes containing equivalent densities of objects offer equivalent opportunities for the use of rat odor cues because the objects they contained were made of the same materials, so the selective pattern of dissociation obtained also rules out explanations in terms of scent marking by the rats. If rats could use unintended odor to perform under our conditions, we would predict that they would do just as well with large as with small boxes, regardless of whether or not they contain objects.

\section{Relationship to Previous Results}

The demonstration that hippocampectomized rats can perform as well as controls on a DNMS task in which small goal boxes containing 3-D objects are used as stimuli, whereas the same rats are impaired when large empty goal boxes are used, is consistent with previous findings from this laboratory (Cassaday \& Rawlins, 1995; Rawlins et al., 1993; Yee \& Rawlins, 1994). A dissociation on this basis also accounts for the apparent discrepancies between the results reported from other laboratories (see above). The difference between small complex boxes and large empty boxes does not depend upon the complexity of the small boxes: Fornicotomized rats chose as accurately as controls when small, empty boxes were used, whereas the same rats showed an impairment when large, empty boxes were used (Cassaday \& Rawlins, 1995). Thus, the presence or absence of objects in the small boxes is unimportant: We suggested that a small goal box is itself treated as an object. However, the question of whether placing objects in large boxes to increase their complexity would differentially aid fornicotomized subjects was left largely unaddressed. The present study (Experiment 2) showed conclusively that when the small boxes' surface decoration and arrangements of objects were reproduced in the large boxes, HIPP rats still chose more accurately with the former than with the latter, although the performance impairment was reduced.

\section{Test of the Associative Distance Account}

We previously proposed that "it seems possible that the rats with lesions fail to use cues flexibly if those cues are associatively distant from the outcome of the response" (Cassaday \& Rawlins, 1995, p. 604). On this analysis, when objects are spread out over the length of one of the large goal boxes, there is spatial discontiguity between the objects on whose basis the choice is made and the objects associated with the outcome of a choice. The subjects would have to bridge this associative gap to perform accurately on the task. This possibility was directly addressed in Experiment 3 of the present study, in which a line of objects extended for the full length of the goal-box floor. In one condition, the line consisted of a series of identical objects, whereas in the other condition it was a series of different objects. In the former, but not the latter, the object seen at the choice point would look identical to that seen at the endpoint. Thus there would be no need to bridge spatial discontiguity in the former group; the latter group controlled for the density of the object display as such.

The results were unambiguous. HIPP rats chose more accurately not when given an associative bridge, but when given a line of distinctively different objects. This is inconsistent with the associative distance account outlined above. Instead, it suggested the possibility that the salience of the object display might be crucial. This assumes that the denser the distribution and the more varied the collection of objects in a display, the more salient that display is. An alternative way to keep salience levels of objects high might be to ensure that the objects are unfamiliar. In Experiment 4, large boxes with objects were used in a PTU schedule so that each correct choice box was seen only once in a session, and such sessions occurred only on alternate days. Under these conditions, HIPP rats showed no sign of a deficit in choice accuracy, even though they still showed a highly significant deficit when tested with large empty boxes.

\section{An Account in Terms of Object-Encoding}

The results of Experiments 3 and 4, taken together, are consistent with the possibility that goal boxes containing objects can be encoded in either of two different ways. One way is to encode them in terms of objects; the other is to encode them in terms of contexts or environments (cf. Rawlins et al., 1993). The tendency to encode as one or the other presumably varies as a function of box size, because, at the extremes, a box can be made so small that it must be encoded as an object, and a box that is large enough becomes an environment like a testing room. Although the whole rat will fit into one of the small boxes (our choice criterion was that the rat should place at least three paws simultaneously on the goal box), very little space remains. Our large boxes are 8 $\mathrm{cm}$ longer than the small boxes, and this difference happens to correspond to one rat body length. We conjecture that this amount of space is psychologically significant to the rat.

Once a box is big enough to be encoded as a context, the 
provision of numerous, salient, discrete objects within the box increases the likelihood that it will nonetheless be encoded in terms of the objects it contains. For controls, either form of encoding can guide appropriate flexible responding so that it makes little or no difference whether the goal boxes are large or small, empty or complex. For rats with hippocampal aspiration lesions, flexible responding depends on the use of encoding in terms of objects.

When the goal boxes are large, we suggest that hippocampal rats cannot encode them as objects, and the presence or absence of discrete objects within the goal boxes can make a difference. But when objects are highly familiar or sparsely distributed, the tendency to encode a goal box in terms of objects will be reduced. This account implies that HIPP rats identify the goal box and its contents as the relevant discriminanda, and that the explicit object cues and the diffuse box cues compete in their control of behavior. If the goal box is relatively large and the objects are few and familiar, then encoding in terms of the context is likely, so that appropriate, flexible responding will not be seen. Note that for any account of this kind to succeed, the rats must discriminate the goal boxes from the goal arms into which they are placed. The goal boxes, as well as the objects they may contain, must be treated as stimuli to which the nonmatching rule applies, whereas the goal arms must be treated as being irrelevant. Otherwise there would be no difference between the size of the context in which objects in small goal boxes are embedded (inside a small box contained within a goal arm context) and the size of the context in which objects in large boxes are embedded (inside a large box contained within a goal arm context). This is a reasonable assumption because in nonspatial DNMS, the experimental procedure ensures that the goal arms are irrelevant: The (relevant) goal boxes move between the sample and choice run on half of the trials and the rat is required to go to the box that is different, irrespective of position.

To develop this account, it would be possible to assess the allocation of conditioning between box cues and objects by studying matching task performance using distinctive large boxes containing differing objects and investigating which cue is dominant in controlling behavior. This could be accomplished by using probe trials following successful acquisition (cf. Clayton, 1995), or throughout acquisition, one group could be required to choose goal box consistently and the other required to choose the particular objects consistently. We predict that hippocampal lesions would impair flexible responding to the diffuse cues provided by the goal boxes but not to the discrete (object) cues.

The object encoding account should also predict that a pair of large boxes, each containing only a single object, should support accurate DNMS performance in rats with hippocampal lesions if the objects are highly salient. However, although we can be precise about novelty, there would be a difficulty in quantifying salience for these purposes a priori.

\section{Object-Encoding and Previous Results}

Our previous study did not show effects of stimulus repetition as such, only effects of goal-box size (Cassaday \&
Rawlins, 1995). Thus it is already established that repetition of the goal-box stimuli in use is not sufficient for a lesion-induced impairment to be seen with small goal boxes. The fact that the relative complexity of the small boxes also made no difference is consistent with the possibility that they are encoded in such a way that little interference is generated. We presume that, when small enough, they are encoded as entities because it makes no difference whether they contain objects or not, so the (non-)matching rule is not necessarily applied to objects within the boxes; the boxes are the objects. But we have now found that the presence or absence of objects can make a difference when the boxes are large. We have suggested that when boxes are large they are not encoded as objects, but they may be encoded as contexts (Cassaday \& Rawlins, 1995; Rawlins et al., 1993). Hence the presence or absence of discrete objects within them could respectively facilitate or preclude any object encoding, in parallel with encoding of the large box itself as a context or environment.

The present results suggest that the novelty or salience of the object array can mitigate against the DNMS impairment seen in hippocampal rats when large goal boxes are used. In Experiment 3, the HIPP group was clearly impaired when large boxes containing a line of identical objects were used, but the HIPP group was not significantly impaired with large boxes containing a line of different objects. This might imply simply that complexity of the array is the critical factor. But in Experiment 4, the HIPP group showed good performance when tested with PTU stimuli that were no more complex than the stimuli that were associated with impaired performance when used repeatedly in Experiments $2 \mathrm{C}$ and 3 . Stimulus novelty could account for the results from all of these experiments; stimulus complexity as such could account only for the results of Experiment 3. On this account, our previous failure to attenuate the DMS deficit in fornicotomized rats tested with large goal boxes by placing familiar objects in the goal boxes stemmed both from object familiarity and from our repeated use of the objects within the test sessions (see footnote 2 in Cassaday \& Rawlins, 1995).

The effects of stimulus novelty seem consistent with earlier studies in which the effects of hippocampal and hippocampus plus amygdala lesions were very mild when large pools of objects were used as stimuli (Aggleton et al., 1989, 1986; Mumby et al., 1992), because these trial-unique schedules ensure a high degree of stimulus novelty on every trial. But in the present study, stimulus novelty was only important when the stimuli were located within a large goal box. Stimulus novelty was unimportant in the small goal boxes because when they were presented repeatedly, there was no effect of hippocampal lesion.

\section{Relationship to Contemporary Theories of Hippocampal Function}

We have previously considered how current theories of hippocampal function can account for the results from our experiments on DMS and DNMS performance in rats with damage to the fornix or hippocampus (Cassaday \& Rawlins, 1995; Rawlins et al., 1993; Yee \& Rawlins, 1994). In 
fairness to those theories of hippocampal function unable to accommodate all of our present findings, we again note that further work with neurotoxic hippocampal lesions may qualify the conclusions reached here.

We previously rejected exclusively spatial theories, but we found that nonspatial theories could provide at least partial accounts of our findings. Does that conclusion still hold? Among the nonspatial theories that we considered at that time, the temporal discontiguity theory (Rawlins, 1985) is inconsistent with our present data, because we found no role for associative distance. The other nonspatial theories can account for some features of the present findings (in particular, the effects of using novel stimuli), but all suffer from a common shortcoming. Kesner's (1991) theory emphasized that an intact hippocampus is needed to encode spatial-temporal information. The present results do in part conform to this pattern, but it is not clear why this theory would predict a deficit when object cues are available, but within a large rather than a small goal box (see Figure 1 [middle]). Exactly the same problem applies to the relational hypothesis of hippocampal function (Eichenbaum et al., 1994; Rawlins, Deacon, Yee, \& Cassaday, 1994) and to the configural theory (Sutherland \& Rudy, 1989, 1995). This latter theory also has a particular difficulty in accounting for the difference in performance with large and small boxes containing identical configurations of objects.

The fact that the contexts provided by the large goal boxes regularly switch their relationship to the rest of the environment should prevent the use of cognitive maps (Cassaday \& Rawlins, 1995, p. 604; O'Keefe \& Nadel, 1978, p. 95), but both cognitive mapping and context learning require processing of configurations or relations among multiple stimuli. Although this common requirement for relating or configuring is consistent with some accounts of hippocampal function, it remains true that neither the relational (Eichenbaum et al., 1994; Rawlins et al., 1994) nor the configurational theory (Sutherland \& Rudy, 1989, 1995) would specifically predict the deficit observed when object cues are available within a large rather than a small goal box or when large rather than small goal boxes are used repeatedly (see Cassaday \& Rawlins, 1995, pp 604-605).

The exclusively spatial cognitive map theory (O'Keefe \& Nadel, 1978) appears to stumble at just the same hurdle as the nonspatial theories. Hippocampectomized rats would surely be expected to show a reduced use of spatial cues, so that such rats' sensitivity to the provision of contextualspatial cues in addition to object cues must be seen as surprising. Finally, Nadel and Willner (1980) likewise predicted that animals with hippocampal lesions should be dominated by cue strategies because place strategies will not work for them. But our data indicate that when diffuse box cues are present, hippocampal rats may fail to make use of any cue-based strategies available to them; moreover, the provision of object cues does not necessarily support a full recovery of DNMS performance. This problem could be resolved for both spatial and nonspatial theories if spatial and nonspatial attributes compete to control behavior even in hippocampectomized rats: This is considered further below.

\section{Conclusions and Implications}

We conclude that novelty promotes the use of objectmediated strategies by hippocampectomized rats: A line of distinctively different objects supports DNMS performance with large boxes, but a line of repeating objects does not; a PTU schedule allows full recovery of performance. We therefore predict that hippocampal rats should generally make use of new stimuli when these are available in sensitive tasks (otherwise associated with a deficit) and spatial deficits should be most apparent with familiar stimuli (cf. Xavier, Stein, \& Bueno, 1990).

We further conclude that large boxes may be treated as whole environments rather than as discrete, large objects partly because of their size as such, and partly because the simple, diffuse nature of the cues provided resembles that typically provided by so-called contextual stimuli (see also Nadel \& Willner, 1980; O'Keefe \& Nadel, 1978, p. 75). On this account, boxes are potentially encoded in two ways: In terms of (a) their spatial attributes (as contexts or environments or as places) and (b) their object attributes. These routes to encoding may compete according to their relative salience (cf. Nadel \& Willner, 1980; Restle, 1957). We have previously suggested that normal rats can respond flexibly to either kind of cue; hippocampectomized rats can respond flexibly to object cues only (Cassaday \& Rawlins, 1995; Rawlins et al., 1993).

We now propose that even hippocampectomized rats, with a deficit in using spatial attributes of stimuli, may nonetheless show impaired encoding of objects when spatial cues are simultaneously present as an alternative source of information. This provides a parsimonious account of why a given configuration of objects and paint finishes can support DNMS performance when displayed in a small box and not when displayed within a larger box (see Figure 1 [middle]). The suggestion that the presence or absence of spatial cues affects the performance of hippocampectomized rats that also have nonspatial cues available is novel. But it finds some resonance in conclusions drawn from discrimination learning experiments: Winocur and Olds (1978) reported that hippocampal rats are particularly affected by changes in context, consistent with the view that contextual cues contributed unusually strongly to behavior in the lesioned group. This finding contrasts with the more widespread view that hippocampal damage prevents animals from using context (e.g., Hirsh, 1974; Phillips \& LeDoux, 1992; Selden, Everitt, Jarrard, \& Robbins, 1991). Until now, in maze tasks, it appears to have been tacitly assumed that hippocampectomized rats simply ignore spatial stimuli, because such rats sometimes show an advantage in learning nonspatial cue variants. In contrast, we conclude that, for these rats, spatial cues, although useless, can nonetheless be profoundly disruptive.

\section{References}

Aggleton, J. P. (1985). One trial object recognition by rats Quarterly Journal of Experimental Psychology, 37B, 279-294.

Aggleton, J. P., Blindt, H. S., \& Rawlins, J. N. P. (1989). The effects of amygdaloid and combined amygdaloid-hippocampal lesions 
upon object recognition and spatial working memory. Behavioral Neuroscience, 103, 962-974.

Aggleton, J. P., Hunt, P. R., \& Rawlins, J. N. P. (1986). The effects of hippocampal lesions upon spatial and non-spatial tests of working memory. Behavioural Brain Research, 19, 133-146.

Cassaday, H. J., \& Rawlins, J. N. P. (1995). Fornix-fimbria section and working memory deficits in rats: Stimulus complexity and stimulus size. Behavioral Neuroscience, 109, 594-606.

Clayton, N. S. (1995). Development of memory and the hippocampus: Comparison of food-storing and nonstoring birds on a one-trial associative memory test. Journal of Neuroscience, 15, 2796-2807.

Deacon, R. M. J., \& Rawlins, J. N. P. (1995). Effects of aspiration lesions of hippocampus or overlying neocortex on concurrent and configural object discriminations in rats. Behavioural Brain Research, 77, 165-174.

Eichenbaum, H., Otto, T., \& Cohen, N. J. (1994). Two functional components of the hippocampal memory system. Behavioral and Brain Sciences, 17, 449-518.

Gaffan, E. A., \& Woolmore, A. L. (1996). Complex visual learning by rats. Learning and Motivation, 27, 375-399.

Herremans, A. H. J., Hijzen, T. H., \& Slangen, J. L. (1995). The object delayed non-matching-to-sample task in rats does not depend on working memory. Neuroreport, 6, 1963-1965.

Hirsh, R. (1974). The hippocampus and contextual retrieval of information from memory: A theory. Behavioral and Neural Biology, 12, 421-444.

Iversen, I. H. (1993). Acquisition of matching-to-sample performance in rats using visual stimuli on nose keys. Journal of the Experimental Analysis of Behavior, 59, 471-482.

Jagielo, J. A., Nonneman, A. J., Isaac, W. L., \& Jackson-Smith, P. A. (1990). Hippocampal lesions impair rats' performance of a non-spatial matching-to-sample task. Psychobiology, 18, 55-62.

Kamil, A. C., \& Sacks, R. A. (1972). Three-configuration matchingto-sample in the pigeon. Journal of the Experimental Analysis of Behavior, 17, 483-488.

Kesner, R. P. (1991). The role of the hippocampus within an attribute model of memory. Hippocampus, 1, 279-282.

Leaton, R. N., \& Borczcz, G. S. (1990). Hippocampal lesions and temporally chained conditioned stimuli in a conditioned suppression paradigm. Psychobiology, 18, 81-88.

Mumby, D. G. (1995). Assessing working memory for objects in rats: No one said it was easy. Neuroreport, 6, 1960-1962.

Mumby, D. G., Wood, E. R., \& Pinel, J. P. (1992). Objectrecognition memory is only mildly impaired in rats with lesions of the hippocampus and amygdala. Psychobiology, 20, 18-27.

Nadel, L. (1991). The hippocampus and space revisited. Hippocampus, 1, 221-229.

Nadel, L., \& Willner, J. (1980). Context and conditioning: A place for space. Physiological Psychology, 8, 218-228.

O'Keefe, J., \& Nadel, L. (1978). The hippocampus as a cognitive map. London: Oxford University Press.

Olton, D. S., Becker, J. T., \& Handelmann, G. E. (1979). Hippocampus, space and memory. Behavioral and Brain Sciences, 2, 315-365.

Olton, D. S., \& Feustle, W. A. (1981). Hippocampal function required for non-spatial working memory. Experimental Brain Research, 41, 380-389.

Paxinos, G., \& Watson, C. (1986). The rat brain in stereotaxic coordinates (2nd ed.). London: Academic Press.

Phillips, R. G., \& LeDoux, J. E. (1992). Differential contribution of amgydala and hippocampus to cued an contextual fear conditioning. Behavioral Neuroscience, 106, 274-285.
Raffaele, K. C., \& Olton, D. S. (1988). Hippocampal and amygdaloid involvement in working memory for non-spatial stimuli. Behavioral Neuroscience, 102, 355-369.

Rawlins, J. N. P. (1985). Associations across time: The hippocampus as a temporary memory store. Behavioral and Brain Sciences, 8, 479-496.

Rawlins, J. N. P., \& Bennett, R. C. (1980). A headholder for visually guided surgery in rats. Physiology and Behavior, 34, $415-416$.

Rawlins, J. N. P., Deacon, R. M. J., Yee, B. K., \& Cassaday, H. J. (1994). Does it still make sense to develop a declarative memory theory of hippocampal function? [Commentary]. Behavioral and Brain Sciences, 17, 492-493.

Rawlins, J. N. P., Lyford, G. L., Seferiades, A., Deacon, R. M. J., \& Cassaday, H. J. (1993). Critical determinants of nonspatial working memory deficits in rats with conventional lesion of hippocampus or fornix. Behavioral Neuroscience, 107, 420-433.

Rawlins, J. N. P., \& Tsaltas, E. (1983). The hippocampus, time and working memory. Behavioural Brain Research, 10, 233-262.

Restle, F. (1957). Discrimination of cues in mazes: A resolution of the "place-vs.-response" question. Psychological Review, 64, 217-228.

Rothblat, L. A., \& Kromer, L. F. (1991). Object recognition memory in the rat: The role of the hippocampus. Behavioural Brain Research, 42, 25-32.

Selden, N. R. W., Everitt, B. J., Jarrard, J. E., \& Robbins, T, E. (1991). Complementary roles for the amygdala and hippocampus in aversive conditioning to explicit and contextual cues. Neuroscience, 42, 335-350.

Shaw, C., \& Aggleton, J. P. (1993). The effects of fornix and medial prefrontal lesions on delayed non-matching-to-sample by rats. Behavioural Brain Research, 54, 91-102.

Sidman, M. (1992). Adventitious control by the location of comparison stimuli in conditional discriminations. Journal of the Experimental Analysis of Behavior, 58, 173-182.

Steele, K., \& Rawlins, J. N. P. (1989). Rats remember long lists of nonspatial items. Psychobiology, 17, 450-452.

Sutherland, R. J., \& Rudy, J. W. (1989). Configural association theory: The role of the hippocampal formation in learning, memory, and amnesia. Psychobiology, 17, 129-144.

Sutherland, R. J., \& Rudy, J. W. (1995). Configural association theory and the hippocampal formation: An appraisal and reconfiguration. Hippocampus, 5, 375-389.

Winocur, G., \& Olds, J. (1978). Effects of context manipulation on memory and reversal learning in rats with hippocampal lesions. Journal of Comparative and Physiological Psychology, 92, 312-321.

Worden, R. P. (1992). Navigation by fragment fitting: A theory of hippocampal function. Hippocampus, 2, 165-188.

Xavier, G. F., Stein, C., \& Bueno, O. F. A. (1990). Rats with dorsal hippocampal lesions do react to new stimuli but not to spatial changes of known stimuli. Behavioral and Neural Biology, 54, 172-183.

Yee, B. K., \& Rawlins, J. N. P. (1994). The effects of hippocampal formation ablation or fimbria-fornix section on performance of a non-spatial radial arm maze task by rats. Journal of Neuroscience, 14, 3766-3774.

Received October 8, 1996

Revision received April 3, 1997

Accepted April 24, 1997 . 